\title{
e-Phaïstos
}

e-Phaïstos

Revue d'histoire des techniques / Journal of the history

of technology

VI-1 2017 | 2018

Varia

\section{La colonie industrielle de Pont-Salomon (Haute- Loire) ou la matérialisation du fouriérisme de Pierre Frédéric Dorian}

The industrial colony of Pont-Salomon or the materialization of the Fourierism

of Pierre Frédéric Dorian

Luc ROJAS

\section{OpenEdition}

Journals

Édition électronique

URL : http://journals.openedition.org/ephaistos/2552

DOI : 10.4000/ephaistos.2552

ISSN : 2552-0741

Éditeur

IHMC - Institut d'histoire moderne et contemporaine (UMR 8066)

Référence électronique

Luc ROJAS, « La colonie industrielle de Pont-Salomon (Haute-Loire) ou la matérialisation du

fouriérisme de Pierre Frédéric Dorian », e-Phaïstos [En ligne], VI-1 2017 | 2018, mis en ligne le 27 juin

2018, consulté le 02 mai 2019. URL : http://journals.openedition.org/ephaistos/2552 ; DOI : 10.4000/ ephaistos. 2552

Ce document a été généré automatiquement le 2 mai 2019.

Tous droits réservés 


\title{
La colonie industrielle de Pont- Salomon (Haute-Loire) ou la matérialisation du fouriérisme de Pierre Frédéric Dorian
}

\author{
The industrial colony of Pont-Salomon or the materialization of the Fourierism \\ of Pierre Frédéric Dorian
}

Luc ROJAS

1 Au milieu de la campagne vellave, à vingt-cinq kilomètres de Saint-Etienne, une colonie industrielle qui s'étend sur trois kilomètres s'offre à notre regard. Le village-usine de Pont-Salomon situé au sein de la vallée de la Semène, affluent de la Loire long de 45 kilomètres, se consacre depuis 1838 à la fabrication des faux et des faucilles. L'homogénéité du lieu prend forme au milieu du XIX ${ }^{e}$ siècle avec l'arrivée des métallurgistes stéphanois qui cherchent les moyens de diversifier leur production. Pierre-Frédéric Dorian, en 1857, achève ce processus en réunissant toutes les unités de production situées le long de la Semène.

2 Homme politique et grand métallurgiste Dorian, au sein de la campagne vellave, érige un projet industriel dans lequel s'exprime en partie sa pensée fouriériste. Inspiré par l'action au Texas de son ami Victor Considérant, Dorian tente de concilier à Pont-Salomon efficacité industrielle et vision sociale. Travaillant pour le monde agricole son usine de faux et de faucille réalise partiellement le dessein de Fourier d'une industrie au service de l'agriculture. Le succès économique, auquel Fourier donne un rôle central dans la réalisation de son idéal, permet l'édification d'une colonie possédant son propre système social et éducatif. L'esprit fouriériste insufflé par Dorian lui survit, ses successeurs pérennisant l'entreprise et la colonie. L'industrie de la faux et de la faucille devient l'acteur majeur de la vallée de la Semène en apportant les progrès liés à l'industrialisation: modernité technique, sociale mais également politique l'usine ayant 
grandement participé à l'émancipation de la commune de Pont-Salomon qui voit le jour en 1865 .

3 Ces grandes lignes retraçant le développement de la colonie industrielle de Pont-Salomon véhiculent paradoxe et contradiction. Comment une communauté formée pour vivre à travers un idéal qui habituellement favorise les comportements autarciques peut-elle connaître la modernité souvent synonyme d'ouverture et d'échange? Quels sont les vecteurs au sein de cette aventure industrielle qui nourrissent ce paradoxe et qui expliquent le succès et l'évolution de la colonie?

\section{De la genèse du site aux établissements Dorian- Holtzer-Jackson ou l'arrivée des industriels stéphanois à la campagne}

4 La fabrication des faux et des faucilles n'est pas une activité traditionnelle en France au début du XIX ${ }^{e}$ siècle. La bordure Est du Massif Central n'échappe pas à la règle. Ainsi il faut attendre 1830 pour voir l'installation, à Saint-Etienne, par Véry d'une usine de faux ${ }^{1}$. Cependant, c'est avec l'arrivée de Nicolas-Alexis Massenet que cette activité commence à prendre un certain essor.

5 En 1816, à Toulouse, est créée la première fabrique de faux et faucilles en acier, Massenet en est le directeur. Malgré l'ambition de pénétrer sur ce marché détenu pas les allemands et les autrichiens, les produits toulousains n'atteignent pas la qualité des faux autrichiennes notamment celles provenant de Styrie. Massenet pense que l'utilisation de l'acier fondu pourrait permettre une augmentation qualitative déterminante de la production. C'est une des raisons qui, à la fin des années 1830, le pousse à venir s'installer à Saint-Etienne. En effet la région stéphanoise est la première en France à produire de l'acier fondu à travers l'usine de l'anglais James Jackson. Massenet rencontre, dans les années 1820 , l'un des fils de Jackson et se lie d'amitié avec lui ${ }^{2}$. Celui-ci fond également du métal et informe Massenet des opportunités qu'offre la région stéphanoise pour la fabrication de faux. Néanmoins il ne s'agit pas de l'unique cause de l'installation de Massenet à Saint-Etienne. Il désire également s'éloigner de l'usine désormais concurrente du Saut-du-Tarn, utiliser le charbon de terre de la région stéphanoise, profiter de la toute nouvelle ligne de chemin de fer qui pourra permettre d'écouler la production ${ }^{3} .$.

En 1837 Massenet s'implante dans le quartier de la Terrasse au Nord de Saint-Etienne et s'associe au soyeux Jean-Baptiste David. Rapidement la société évolue, le 30 avril 1840 une nouvelle entreprise se forme entre MM. Massenet, Guillaume Gerin (quincaillier) et William Jackson ${ }^{4} .1842$ marque un tournant dans la fabrication des faux dans le bassin stéphanois. Cette année là, l'usine de la Terrasse est à même de fabriquer 100 à 130000 faux par an soit une production conséquente permettant une exportation en direction de la Suisse ${ }^{5}$. Malgré ce succès la concurrence apparaît. Durant l'année Pierre-Frédéric Dorian crée une usine de faux dans la vallée du Furan près de Rochetaillée au lieu-dit « les Balaires» et Chalayer, au sein de la proche vallée de l'Ondaine, s'installe comme concurrent potentiel. Malgré le succès commercial naissant, la société " Massenet, Gerin et Jackson » investit progressivement un nouveau lieu de production. Entre 1842 et 1845, l'entreprise achète diverses chutes d'eau et propriétés sur le cours de la Semène : c'est le début de l'installation à Pont-Salomon' . 
$7 \quad$ Il est intéressant de remarquer que ce sont les grands métallurgistes stéphanois, Jackson et Dorian notamment, qui s'attèlent au développement d'une telle industrie. La production de faux et de faucilles, autrement dit d'outils agricoles, est un excellent moyen pour ces industriels de diversifier leurs débouchés et de minimiser les effets des crises métallurgiques. Il faut noter que les statuts de ces fabriques de faux prévoient la fourniture exclusive des aciers. Pour la société « Massenet, Gerin et Jackson », c'est la maison Jackson qui dispose de ce privilège ${ }^{7}$. Plus tard lorsque l'entreprise sera alliée à Dorian, les statuts prévoiront la fourniture des aciers ainsi: "Jackson frères, Petin, Gaudet et Cie » fournissent les deux tiers des aciers et « Jacob Holtzer ${ }^{8}$ et Cie » un tiers. Le site de Pont-Salomon offre un avantage notable. Les concurrents emploient pour la plupart des moteurs hydrauliques, pour se trouver dans les mêmes conditions, il faut user des mêmes moyens. En s'installant au bord de la Semène " Massenet, Gerin et Jackson » s'offre une force de 2 à 300 chevaux, que l'on peut encore accroître en utilisant de nouvelles chutes. D'un autre côté Pont-Salomon n'est qu'à sept kilomètres de Firminy dont le prix du charbon est plus avantageux qu'à Saint-Etienne9 .

En octobre 1856, les concurrents deviennent associés. Après son mariage avec Mlle Holtzer, Dorian reste le seul propriétaire de l'usine de faux des Balaires et décide de s'allier, dans cette activité, avec Massenet, Jackson et Gerin. Le journal le Mémorial de la Loire et de la Haute-Loire annonce, le 7 novembre 1856, la naissance de La Gerbe : société des fabriques de faux et faucilles de Saint-Etienne, Jackson, Gerin, Dorian et $\mathrm{Cie}^{10}$. A partir de cette date la société entreprend la modernisation de ses ateliers. Mais c'est en 1859 avec la mise en activité de l'ensemble industriel dit de l'Alliance que le potentiel de l'entreprise prend une réelle importance. C'est également à cette date que les usines stéphanoises du quartier de la Terrasse sont abandonnées définitivement faisant du site de Pont-Salomon l'unique lieu de production.

10 Pierre Frédéric Dorian prend au sein de cette nouvelle société une place considérable qui s'accroît en 1863 quand la famille Gerin vend ses actions aux familles Holtzer et Dorian faisant de ces dernières les actionnaires majoritaires ${ }^{11}$. Dès cet instant Pierre Frédéric Dorian devient le décisionnaire de la nouvelle société, « Dorian-Holtzer, Jackson et Cie », qu'il modèle à sa pensée. 
Les usines de l'Alliance en 2017

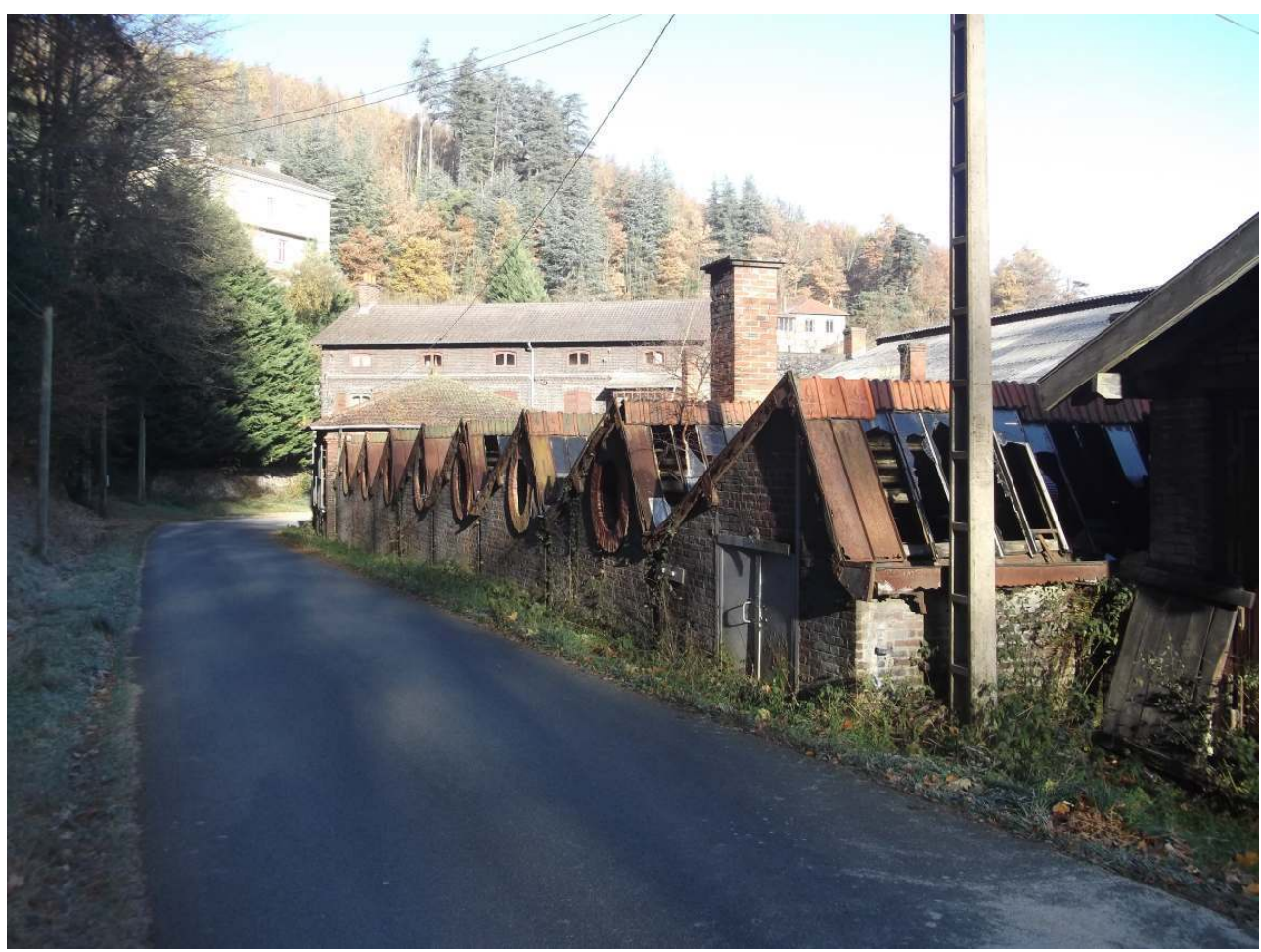

Vue sur les premiers sheds installés dans le bassin industriel stéphanois

Crédit personnel de l'auteur

\section{Les usines de l'Alliance en 2017}

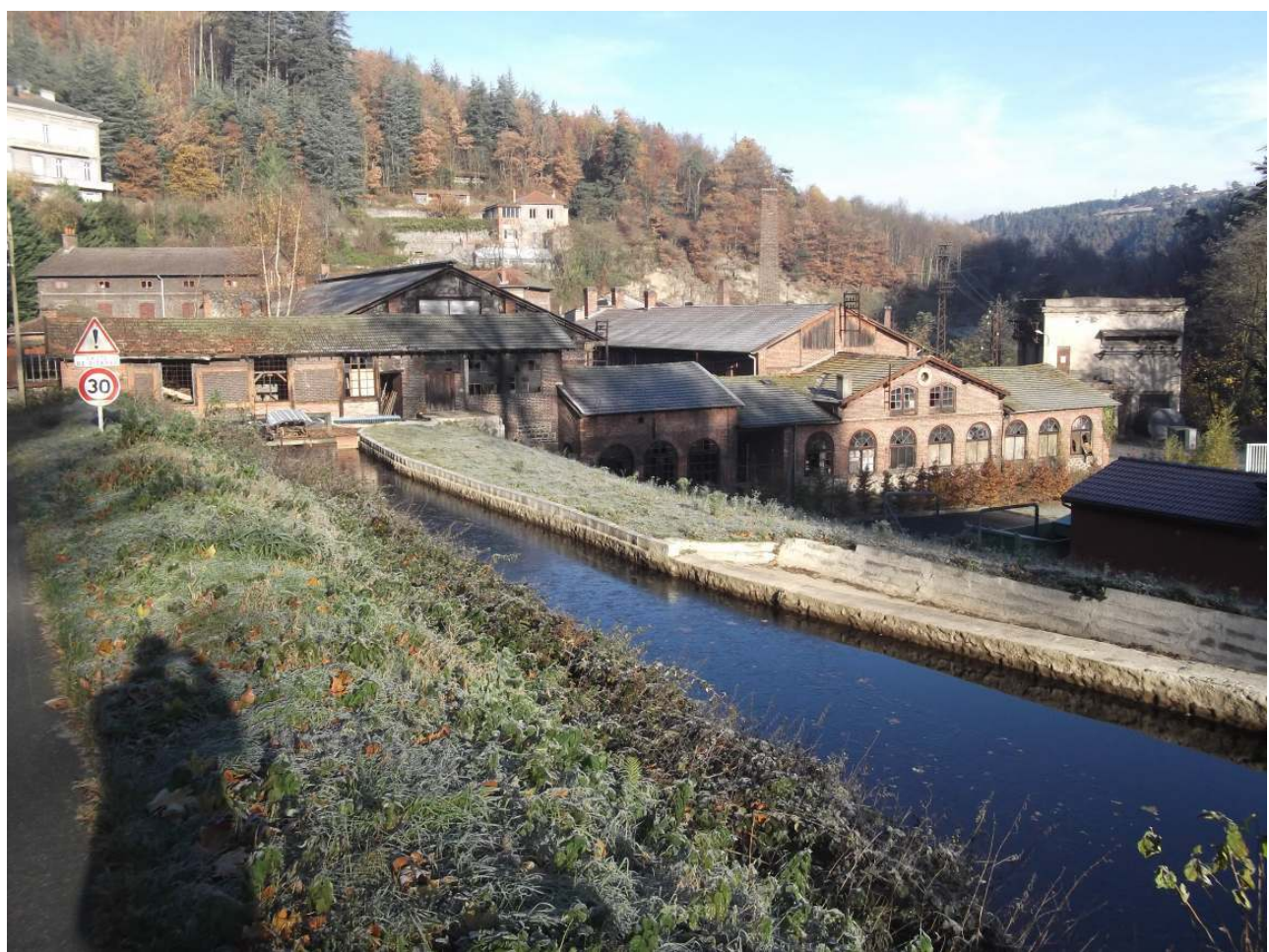

Vue générale

Crédit personnel de l'auteur 


\section{La pensée industrielle sur les bords de la Semène}
de la Semène de la grande industrie métallurgique et de son imposante présence.
Valserres dans son enquête datant de 1862 saisit la taille de la fabrique de faux,
déjà conséquente:
"Les six usines du Pont-Salomon comprennent 12 roues hydrauliques de
force de 180 chevaux, 3 turbines de 75 chevaux, plus de 45 chevaux appliqués force de 180 chevaux, 3 turbines de 75 chevaux, plus de 45 chevaux appliqués
à divers. Ces forces font mouvoir 35 marteaux à queue et neuf machines soufflantes. Ces dernières portent le vent à 35 feux de forge, à 7 fours de releveurs, à 1 four à gaz pour la trempe, à 4 fours de recuisseurs, à 2 fours de bleuisseurs, à 4 feux de forge pour l'entretien du matériel. Enfin, il y a une usine à gaz fournissant 200 becs pour l'éclairage ${ }^{12}$. »

Il est ici question d'un ensemble à mille lieues de l'artisanat que l'on trouve habituellement dans ces zones rurales. Cette société compte, lors de sa naissance, déjà 200 ouvriers. A la fin du XIX siècle, ces établissements font travailler, selon l'inspection du travail, 300 personnes ${ }^{13}$.

L'entreprise dispose, dans ses approvisionnements en matières premières comme dans ses débouchés, de conditions exceptionnelles. Elle est intégrée à un ensemble industriel beaucoup plus vaste, puisque Pierre Frédéric Dorian, gendre de Jacob Holtzer, est depuis 1862 co-directeur des importantes aciéries d'Unieux, à l'Ouest de Saint-Etienne, et Député du département de la Loire à partir de 1863. La fabrique de faux de Pont-Salomon ne constitue qu'un pan de l'empire métallurgique de Dorian.

Les pratiques et les réseaux commerciaux sont ceux de la très grande entreprise. Dès les années 1860 les lieux de vente s'internationalisent: Algérie, Suisse, Italie... La société dispose, dès sa création, d'un réseau très développé en France à la campagne comme à la ville. Elle profite des réseaux des grands industriels stéphanois. On voit, par exemple, apparaître, en 1859, dans les contacts et les clients des noms comme Paravicini originaire de Bâle qui est en affaire avec les rubaniers stéphanois. On assiste aux alentours des années 1880 à une européanisation très ciblée de la clientèle notamment en direction du Nord de l'Espagne et du Nord de l'Italie. Ainsi entre 1880 et 1910, apparaissent des clients séjournant à Bilbao, Saragosse, Alavès, Milan, Turin... ${ }^{14}$ Afin d'investir ces marchés «Dorian-Holtzer, Jackson et Cie » utilise des intermédiaires locaux. En Italie, dans les années 1890, M. Tamagno séjourne à Turin et parcours le Nord de la péninsule afin de démarcher les clients dans les campagnes italiennes. Parallèlement en Espagne, dès 1880, M. Burgell se déplace très souvent entre Barcelone et Valladolid pour aller à la rencontre d'éventuels clients au sein de la campagne espagnole ${ }^{15}$.

La logique industrielle de la grande entreprise métallurgique pousse Dorian à s'adapter au potentiel humain local. Pour fabriquer une faux, il est nécessaire d'employer un personnel très spécialisé. Ainsi le savoir faire détenu au sein de l'entreprise est l'apanage d'ouvriers "déracinés ", autrement dit d'ouvriers spécialement recrutés par la société pour leur savoir-faire. Il convient de remarquer que cette pratique est courante chez les industriels stéphanois dans leur démarche d'innovation technique ${ }^{16}$. Avant même l'implantation à Pont-Salomon, Massenet aurait été en contact et aurait visité les usines de faux de Neuenbuerg (Allemagne) vers 1840 pour négocier avec des ouvriers leur venue 
en France. L'un d'entre eux aurait semble-t-il accepté ${ }^{17}$. Jacques Valserres dans son enquête de 1862 nous présente la colonie de Pont-Salomon comme une mosaïque culturelle. On compte, notamment, trois nationalités majeures: des autrichiens (tyroliens, styriens), des allemands (badois, wurtembergeois) et des français (toulousains, ariégeois, jurassiens $)^{18}$. Cette présence extérieure à la vallée de la Semène est si importante qu'il existe un ressenti négatif de la population locale qui assimile la fabrique de faux à l'Allemagne.

Le site de Pont-Salomon est un argument de choix dans l'implantation de l'usine aux bords de la Semène. Toutefois cet évènement demeure paradoxal. En effet ce sont de grands métallurgistes, donc des tenants de l'énergie vapeur, qui s'installent là et qui utilisent l'énergie hydraulique. A travers ce choix ces industriels effectuent, symboliquement, un retour en arrière puisqu'ils s'exposent aux caprices de la nature et du temps. L'avantage de l'énergie vapeur demeure sa régularité offrant une production très contrôlable. A Pont-Salomon Dorian devient dépendant de l'abondance de l'eau et donc du climat, il n'est pas rare que les usines chôment en cas de relative sécheresse ${ }^{19}$. L'entreprise tente au fil du temps d'atténuer les effets de cette dépendance. Ainsi un rapport de stage nous apprend que la société possède, dans les années 1920, une machine à vapeur de 200 chevaux destinée, en cas de manque d'eau ou d'avarie, à fournir l'énergie nécessaire ${ }^{20}$. Il est également intéressant de remarquer que les métallurgistes stéphanois ont choisi d'investir dans la fabrication de faux afin d'élargir leurs débouchés et de se prémunir contre les crises métallurgiques. Si par cet investissement ils minorent l'importance des dépendances, ils multiplient ces dernières. En effet les récoltes de l'année et l'état de l'agriculture dictent aux agriculteurs leurs besoins en faux offrant ainsi un marché plus ou moins intéressant à « Dorian-Holtzer, Jackson et Cie ».

17 La société construit son complexe technique en s'appuyant, tout d'abord, sur les sites existants édifiés à partir d'anciens moulins de papiers. Mais rapidement afin d'augmenter sa capacité de production l'utilisation de turbine devient incontournable. Le complexe technique de l'entreprise prend une envergure de premier plan au tournant du XXe siècle puisqu'elle décide d'électrifier l'intégralité de ses sites. Afin d'atteindre ce but la réflexion devient globale. Il n'est plus question que chaque unité produise sa propre énergie. Dorénavant des usines génératrices d'électricité vont distribuer la force motrice à l'ensemble des unités de production. Il s'agit évidemment d'hydro-électricité produite par des centrales hydro-électriques situées en amont. En 1902 est édifiée la station Méane I qui profite d'une hauteur de chute de 11,5 mètres produisant $120 \mathrm{Kw} / \mathrm{h}$. La station Méane II est constituée en 1922 près d'une chute de 10,5 mètres offrant $100 \mathrm{Kw} / \mathrm{h}$. Les sites sont tous alimentés par ces usines et connectés à ces dernières par des lignes aériennes ${ }^{21}$. $\mathrm{Ce}$ complexe technique constitue la base de l'usine de faux pendant le reste de son existence. En 1960 celui-ci semble archaïque au regard des moyens de l'époque. En effet sur 205 machines seulement 14 ont été installées après $1925^{22}$.

La logique industrielle s'exprime au sein de l'usine Dorian par une préoccupation constante : obtenir le prix de revient le plus rémunérateur. Dès 1857 des études sont réalisées, tout est pris en compte pour le calcul du prix de revient : acier, bougie, chiffon... La nature des fournitures est très détaillée rien n'échappe à l'analyse ${ }^{23}$. Dans les années 1930 cette démarche devient plus "scientifique», une étude est réalisée pour la fabrication de chaque type de faux. Toutes les opérations de fabrication et de commercialisation sont analysées afin d'obtenir le meilleur prix de revient. Chaque produit est soumis à l'analyse d'un coefficient spécial qui varie en fonction du lieu de 
vente de la faux: vente à l'étranger ou sur place... Conscient de la concurrence l'entreprise effectue des comparaisons entre ses coûts de fabrication et ceux des principaux concurrents ${ }^{24}$.

19 La division et l'organisation du travail, corollaires du prix de revient, constituent des domaines d'études importants pour la société. Dès 1862 Valserres nous fait remarquer :

« Le travail est divisé de manière à ce que le même ouvrier fasse toujours la même opération. Ce système, qui n'existe pas au même degré dans les autres fabriques de faux, contribue beaucoup à la perfection des produits; le travail est plus rapide, le prix de revient moins élevé, et cependant les ouvriers touchent de bons salaires ${ }^{25}$.

Influencé par les mouvements d'organisation scientifique du travail du $\mathrm{XX}^{\mathrm{e}}$ siècle, les usines Dorian lancent de nombreuses études sur le temps de travail. Dans les années 1930, influence du taylorisme oblige, un rapport s'attache à décortiquer les gestes de chaque opération puis recommande par la suite un temps de référence pour la fabrication de chaque type de faux ${ }^{26}$. Après la seconde Guerre Mondiale les études se complexifient. Le temps de travail demeure une préoccupation centrale: chaque geste est calculé en seconde, puis l'on définit le temps moyen d'un cycle permettant la réalisation d'une opération. Le cycle en question correspond à un pourcentage de temps actif par rapport au temps total de travail. L'objectif des établissements Dorian-Holtzer et Jackson est d'augmenter ce temps actif ${ }^{27}$.

21 Cette logique industrielle qui s'installe sur les bords de la Semène engendre un système social qui n'est pas forcément à l'image du reste du monde industriel. En effet l'usine de Pont-Salomon est modelée par Pierre-Frédéric Dorian fouriériste convaincu et actif s'il ont en croit son active correspondance avec Victor Considérant.

\section{Dorian-Holtzer, Jackson et Cie : une utopie fouriériste?}

Pierre-Frédéric Dorian est né à Montbéliard en 1814 où ses parents possèdent une petite forge et un martinet produisant des outils pour l'agriculture. Il arrive dans la région stéphanoise pour des raisons de scolarité puisqu'il effectue une partie de celle-ci à l'École des mines de Saint-Étienne. A la fin de son cursus scolaire il participe à la tentative de la colonie sociétaire fouriériste de Condé-sur-Vesgre puis se lance dans les affaires tout en conservant des liens très importants avec les milieux fouriéristes. Il est notamment l'agent électoral de Victor Considérant.

23 Hormis l'opportunité industrielle, quel attrait peut engendrer Pont-Salomon sur PierreFrédéric Dorian? Le site vellave est comparable à celui de Condé-sur-Vesgre où il a vécu. Il est surtout en accord avec les idées de Fourier: une affinité entre industrie et agriculture, l'industrie produisant pour le monde agricole. Dans Le nouveau monde industriel et sociétaire Charles Fourier insiste sur l'adéquation entre industrie et monde agricole local ${ }^{28}$. Pour lui si une manufacture s'installe dans un lieu c'est avant tout pour apporter une aide aux agriculteurs locaux. Dans ces paysages agricoles escarpés de la bordure Est du Massif Central la faux semble ainsi trouver naturellement sa place.

24 La société «Dorian-Holtzer et Jackson » n'est pas à proprement parler un phalanstère fouriériste. En premier lieu parce que l'association originelle n'est pas une alliance 
philosophique ayant pour but d'appliquer les idées de Fourrier mais une organisation industrielle et économique. Dorian n'est pas totalement libre et doit composer avec ses associés. Toutefois l'entreprise est très fortement marquée, dans son territoire, dans sa politique sociale, par l'utopie fouriériste. L'un des exemples les plus frappants demeure la constitution de l'ensemble industriel dénommé «L'Alliance» construit en 1857 regroupant les ateliers, les logements ouvriers et le logement du directeur. Il faut noter que le reste du site se développe suivant la même dynamique. Il est fort à parier, connaissant les liens unissant Dorian à Considérant, que «L'Alliance ${ }^{29}$ " est constituée en écho à la colonie sociétaire qu'avait tenté d'établir, au Texas, quelques années auparavant, en 1852, Victor Considérant. Celle-ci prenant un nom tout aussi symbolique que «L'Alliance » à savoir « Réunion ».

Dès les premières années une véritable communauté voit le jour. Valserres en 1862 parle de « communauté de forgerons ${ }^{30}$ ». Celle-ci perdure dans le temps. Dans l'après Première Guerre mondiale, des actes notariés nous rappellent que l'immense majorité des habitants de Pont-Salomon y sont nés et ont un lien direct avec l'usine de faux ${ }^{31}$. Pierre-Frédéric Dorian est conscient de fonder une communauté industrieuse au bord de la Semène. S'il en est le fondateur il ne peut se consacrer que de manière relative à cette tâche puisque son empire métallurgique et ses importantes fonctions politiques l'accaparent grandement ${ }^{32}$. Jusqu'à sa mort en 1873, il est épaulé par Fleury Binachon qu'il nomme directeur de l'usine en 1857. Ce dernier apprécie les idées sociales de Dorian et devient en quelque sorte le continuateur de son œuvre. Fleury Binachon exerce les fonctions de directeur jusqu'en 1879 et est remplacé par son fils Joannès qui jusqu'en 1911 fait perdurer, par sa politique sociale, l'esprit fouriériste au sein de l'entreprise ${ }^{33}$.

Cet esprit fouriériste se matérialise tout d'abord par la construction de nombreux logements ouvriers. L'immense majorité des employés sont logés par l'usine à proximité de leur lieu de travail. Chaque groupe de logements est associé à un lieu de production ainsi les ouvriers sont liés à L'Alliance, La Méane, Le Foultier... Le nombre de logements est très important et augmente avec le temps. Un inventaire détenu par l'entreprise, en 1974, évalue la possibilité de loger un peu moins de 2000 personnes $^{34}$. La location d'un appartement est réservée aux employés, il s'agit d'une clause du contrat de travail. Si l'employé est malheureusement renvoyé il doit quitter son logement. A ces appartements loués, pour des sommes modiques, sont toujours associés des jardins également localisés près du lieu de travail. On compte sur les terrains de l'entreprise plus de 200 jardins ${ }^{35}$. Ces derniers ont divers objectifs : améliorer l'ordinaire, offrir un loisir à l'ouvrier et surtout le soustraire à l'oisiveté, thème récurrent dans la pensée fouriériste. 


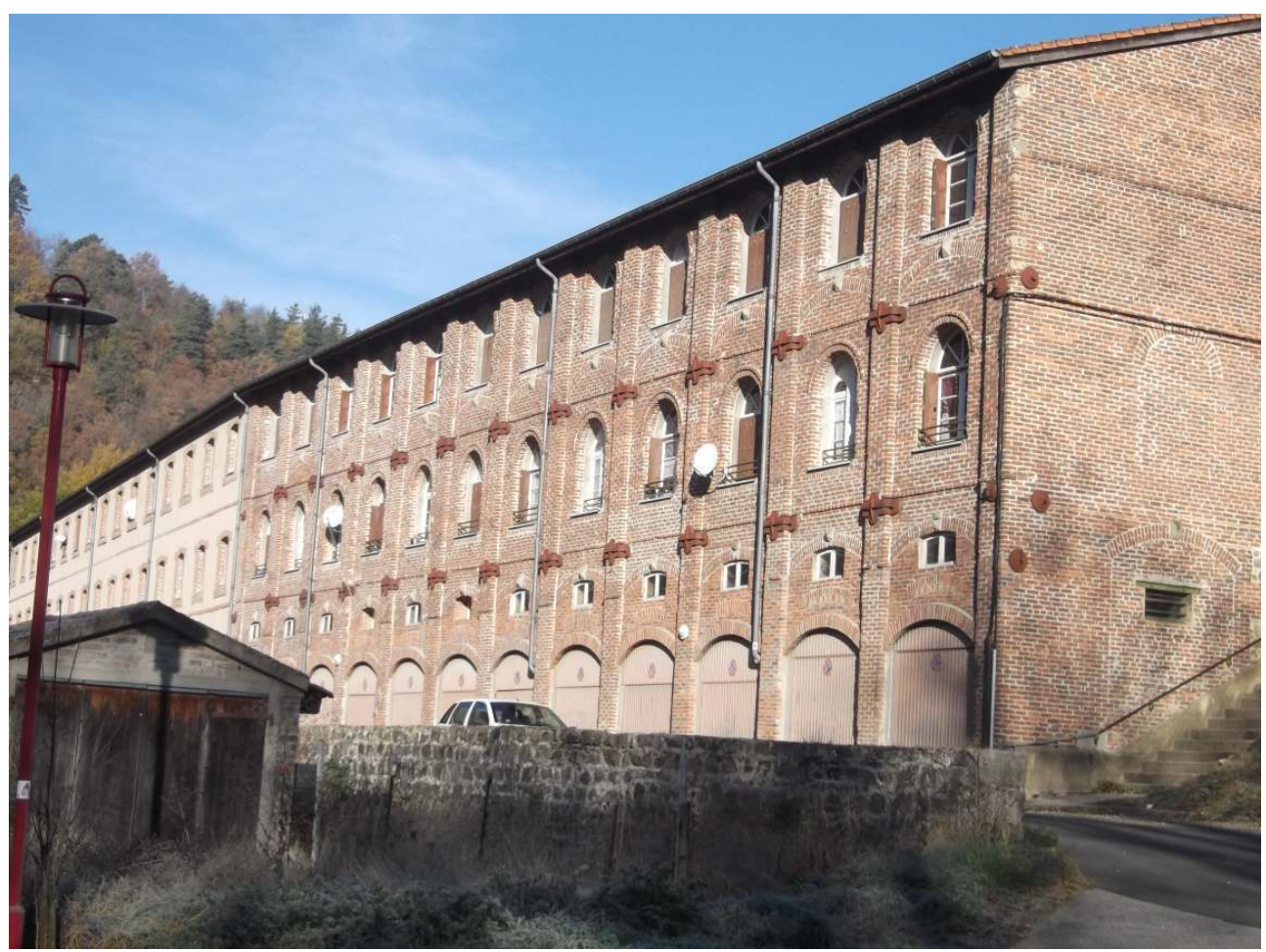

Crédit personnel de l'auteur

La communauté fondée par Dorian est installée sur un territoire appartenant à trois communes différentes, il n'est donc pas apparemment question d'édifier une église sur le site de l'usine de faux. Toutefois n'oublions pas l'importance du culte pour Fourier. Pour lui la religion constitue l'une des trois routes de l'association. Celle-ci sera :

«[...] religieuse par passion, par conviction de la haute sagesse de Dieu, dont elle recueillera à chaque instant les bienfaits. Le culte public sera pour elle un besoin : le moindre vicaire y jouira du sort actuel des évêques, et on sera obligé en France de créer, par ordination accélérée, au moins trente mille prêtres, afin que chaque phalange en ait un nombre suffisant pour exercer en relais, sans assujettissement journalier à leurs fonctions ${ }^{36}$. »

Ainsi Pierre-Frédéric Dorian décide dès les premières années d'édifier une chapelle dans un bâtiment de production où officie l'abbé Viou qui fait également partie de la fanfare de l'entreprise. En 1866, la société propose au Préfet du département un terrain pour construire une église. En 1872, quelques années après la création de la commune de PontSalomon, Dorian fait don à la commune de l'église et du presbytère qui viennent d'être achevés en vue de l'établissement d'une paroisse ${ }^{37}$. 


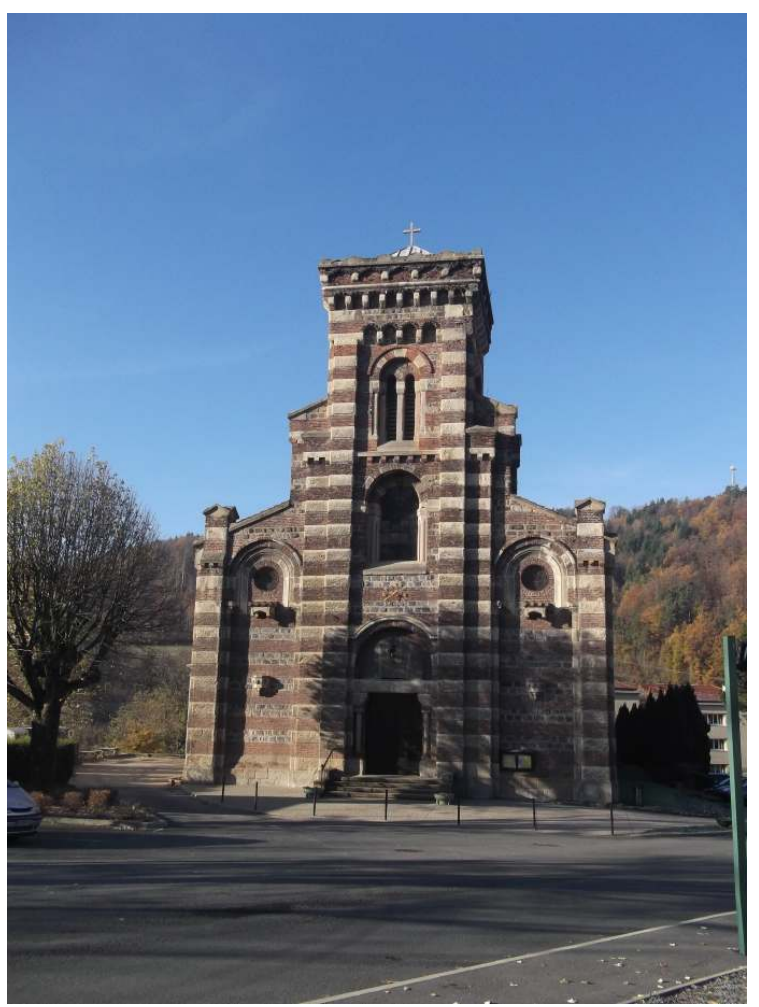

Crédit personnel de l'auteur

\section{Presbytère}

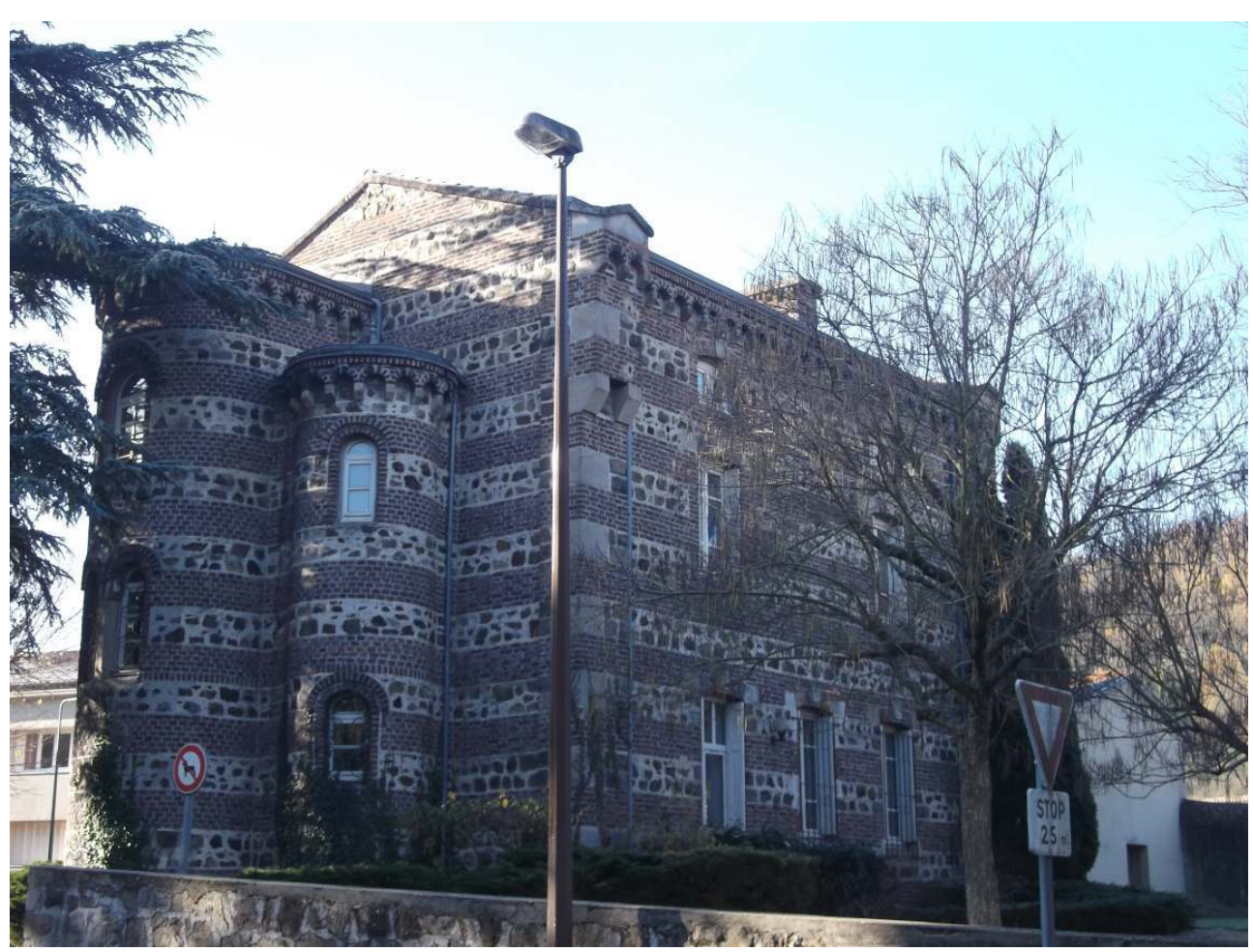

Le bâtiment est réutilisé actuellement comme mairie par la commune de Pont-Salomon crédit personnel de l'auteur 
Dès 1861 est créée une caisse de secours mutuels qui deviendra une société de secours mutuels en 1866. Alimentée par les dons de l'entreprise et par une cotisation des ouvriers en fonction du montant de leur salaire, cette caisse a pour objectif d'assurer une aide médicale aux membres de la communauté. C'est une institution qui se veut juste, morale et qui se place dans une logique sociétaire et fouriériste comme nous le rappelle l'introduction des statuts :

«Secourir et aider ses frères, c'est suivre la loi de Dieu. Si l'association a été reconnue comme le meilleur et le plus puissant moyen pour atteindre ce résultat, c'est surtout parmi les ouvriers d'un même corps ou d'une même manufacture qu'une association mutuelle doit faire ressortir tout ce qu'elle a de puissance, de moralisation et d'heureuse prévoyance. Les ouvriers des fabriques de faulx (sic) de Pont-Salomon l'ont compris, et ils ont constitué, avec l'approbation et sous la surveillance de leur Directeur, la société dont suivent les statuts. ${ }^{38}$

En 1878 sous l'influence de Fleury Binachon est érigé un cercle des ouvriers. Cette institution dirige une part importante des loisirs des ouvriers et notamment les activités destinées à l'instruction personnelle. L'article 1 des statuts du cercle résume le but de cette assemblée :

«Il est institué dans la commune de Pont-Salomon un cercle sous la dénomination de cercle des ouvriers. Son but est de contribuer, par tous les moyens en son pouvoir, à propager les idées fraternelles et de solidarité qui doivent être la base de toute société; d'aider au développement de l'intelligence par la création d'une bibliothèque commune, la lecture des journaux et la démonstration de toutes idées utiles par des lectures ou des conférences qui pourraient être faites par des membres du cercle ou par d'autres personnes qui voudraient bien nous honorer de leurs concours ${ }^{39}$. »

31 A travers cette entité Binachon tente de modeler l'esprit de la communauté en lui inculquant des valeurs et des connaissances communes. Le directeur de l'entreprise recherche une certaine concorde sociale au sein de sa population. D'ailleurs afin de limiter les conflits idéologiques et de tenir écartée la communauté des tensions politiques extérieures l'article 2 des statuts du cercle interdit les discussions politiques. L'administration de l'entreprise veille au respect des statuts afin de tendre au maintien de la bonne harmonie et de la fraternité entre tous les membres du cercle. Le contrôle va jusqu'au choix des livres et des journaux, ces derniers devant traiter surtout d'industrie, d'économie sociale, de la marche générale des société coopératives, d'histoire, de science et d'arts ${ }^{40}$.

32 Cet esprit de communauté distillé par Dorian et Binachon père et fils depuis les années 1860 s'effrite à partir des années 1930 avec l'apparition notamment, en 1936, de deux syndicats l'un ouvrier l'autre patronal au sein des établissements «Dorian-Holtzer, Jackson et Cie $»^{41}$. Malgré cet évènement qui marque l'ouverture intellectuelle et politique de la communauté, cette dernière restera un lieu où l'usine de faux conserve une influence prépondérante sur ce qui est désormais devenue la commune de Pont-Salomon. 


\section{Quand l'industrie devient un vecteur de modernité....}

Préfet en août 1865. Il ne s'agit pas d'un membre de l'entreprise. Fleury Binachon fait pression sur le Préfet lors de l'élection des conseillers municipaux en 1866. Après un imbroglio politique, le résultat est éloquent : Jules Holtzer devient, le 4 novembre 1866, Maire avec pour adjoint Fleury Binachon. A partir de cette date et ce pour plusieurs décennies les maires seront liés ou alliés à l'entreprise de faux. Cette main mise sur l'autorité politique communale facilitera l'activité industrielle de Dorian. Dès 1867 les besoins de déserte ferroviaires se font sentir. Le conseil municipal, acquis à la cause de l'usine Dorian, fait remarquer au Préfet que le chemin de fer demeure d'utilité publique et est un élément de développement pour toutes les usines mues par les eaux de la Semène. Après plusieurs années, en 1885, Pont-Salomon est relié à la nouvelle ligne Firminy-Annonay. Celle-ci est une révolution pour la commune mais surtout pour l'usine qui désormais achemine ses matières premières et ses productions par le biais du train.

Rapidement la nouvelle commune se dote des attributs synonymes d'indépendance et de progrès. En 1886, le conseil municipal demande l'installation d'un bureau de poste qui jusque là se trouve à Saint-Férréol. Un bureau télégraphique s'ouvre en 1886 et est confié au receveur-buraliste de la commune. Cette ouverture est possible grâce à la souscription organisée par l'entreprise Dorian qui à cette époque compte de nombreux correspondants en France et à l'étranger. Malgré tout, la situation ne convient pas au développement de l'usine de faux. La commune étudie au début des années 1930 la possibilité de construire un bureau de poste mais cette éventualité est rapidement abandonnée. C'est une fois de plus « Dorian-Holtzer, Jackson et Cie » qui se substitue à l'autorité publique. M. MartinBinachon, alors sénateur de la Haute-Loire, informe, dans une lettre du 15 avril 1937, la 
direction régionale des postes que l'usine est prête à louer un de ses immeubles à la commune afin d'installer le bureau de poste ${ }^{42}$.

Dès 1902, nous l'avons dit, l'entreprise produit son électricité à partir des centrales hydro-électriques installées au hameau de la Méane ${ }^{43}$. Cette électricité est aussi distribuée au sein de la commune pour l'éclairage public. Avec la construction de la deuxième centrale hydro-électrique la Méane II, ce sont l'intégralité des foyers de Pont-Salomon qui peuvent désormais profiter de «la fée électricité ». Une publication de la société, datant de l'après deuxième Guerre Mondiale, nous présente Pont-Salomon comme la première ville électrique de France ${ }^{44}$.

En 1929 une source d'eau potable, donnant un débit d'environ $4 \mathrm{~m}^{3}$ d'eau à l'heure, est découverte sur les bords de la Semène dans une propriété appartenant à la société. L'emplacement de la source se trouve bien en contre bas du village de Pont-Salomon. L'usine de faux décide d'installer des adductions d'eau pour cela une station de pompage est édifiée. Celle-ci fonctionne grâce au courant électrique qui est amené par une ligne spéciale venant des usines Dorian. A un point haut du village il est établi un réservoir en maçonnerie permettant la distribution de l'eau dans la commune. Cette station assure l'alimentation d'une bonne partie de la commune. Sur l'ensemble de ce réseau de canalisation, quatre bornes fontaines et deux bouches d'incendie sont placés et de nombreux habitants font installer l'eau à leur domicile. Les travaux engendrés par ces adductions d'eau coûtent 300000 francs entièrement payés par l'usine Dorian ${ }^{45}$.

Si la deuxième moitié du vingtième siècle est synonyme de la fin de la communauté fouriériste, l'usine de faux continue à jouer un rôle prépondérant dans la vie du village. Elle possède et mets à la disposition des habitants de Pont-Salomon des équipements collectifs qui sont habituellement du ressort de l'autorité communale. Au détour de travaux effectués au sein de bâtiments appartenant à la société nous apprenons que les usines Dorian possède une salle des fêtes mise régulièrement à la disposition du public ${ }^{46}$. Le cinéma, symbole de la modernité d'après guerre, arrive à Pont-Salomon grâce aux usines Dorian puisque ce sont elles qui installent un cinéma en 1950 au sein du village ${ }^{47}$.

\section{Conclusion}

L'histoire singulière de la colonie industrielle de Pont-Salomon, formée autour des établissements « Dorian-Holtzer, Jackson et Cie ", nous a légué un patrimoine conséquent marquant le paysage de la campagne vellave. Outre les bâtiments liés à la production de nombreuses autres traces demeurent. Celles-ci sont directement liées à l'esprit fouriériste du site : logement, jardins, église, château de l'Alliance... 


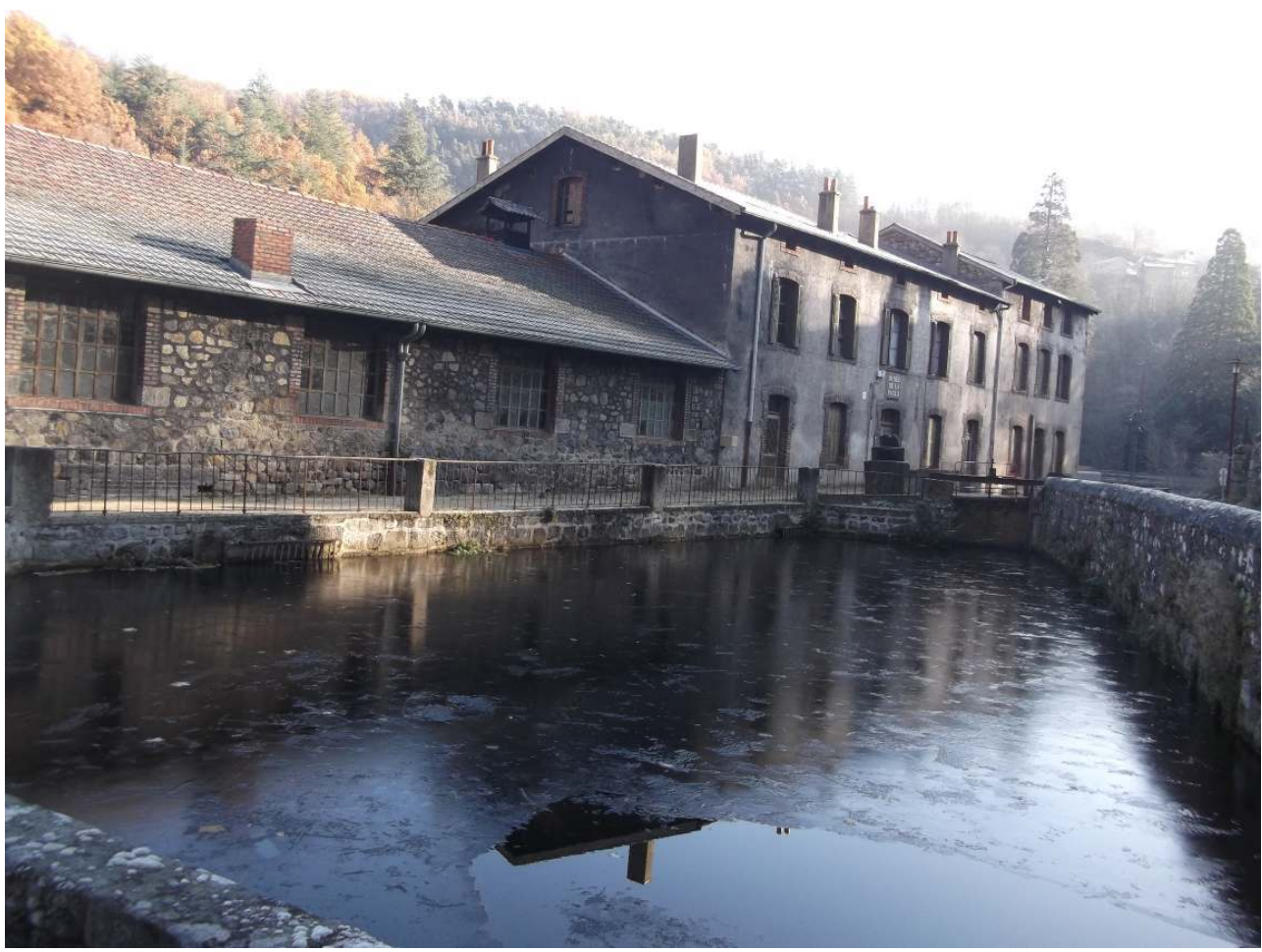

Le site abrite actuellement le musée de la Faux et de la vie ouvrière

crédit personnel de l'auteur

Le site constitue un potentiel patrimonial tout aussi remarquable que la colonie textile Sedo près de Barcelone ou une autre colonie textile fondée par Robert Owen et proche du socialisme utopique, actuellement classée patrimoine mondial de l'humanité par l'UNESCO, à savoir New-Lanark (Écosse). Contrairement à ces deux villages-usines et malgré l'arrêt de la production Pont-Salomon n'a pas connu la désertification temporaire ou définitive des autres villages-usines. En effet, la cité de Haute-Loire est rapidement devenue un village satellite ancré dans la périurbanisation qui se développe dans la seconde moitié du $\mathrm{XX}^{\mathrm{e}}$ siècle au point parfois d'en oublier son histoire.

\section{BIBLIOGRAPHIE}

Anonyme, Les Établissements Dorian-Holtzer-Jackson et Cie, Circa 1970

DESCREUX Denis, Notices Biographiques Stéphanoises, Saint-Étienne, Imprimerie Théolier, 1868

FOURIER Charles, Le Nouveau Monde Industriel et Sociétaire, Paris, Librairie de l'école sociétaire, 1845

JACKSON William, James Jackson et ses Fils, Saint-Étienne, 1883 
ROJAS Luc, L'industrie stéphanoise : de l'espionnage industriel à la veille technologique (1700-1950), Paris, l'Harmattan, 2009

VALSERRES Jacques, Les Industries de la Loire, Saint-Étienne, Robin, 1862

\section{Sources}

Archives départementales (AD) de la Haute-Loire, Fonds Dorian-Holtzer-Jackson.

AD Haute-Loire, 9 M 15, inspection du travail, établissement soumis à l'inspection du travail : liste de la commune de Pont-Salomon (16 août 1897).

AD Haute-Loire, 10 M 36, syndicats professionnels, Pont-Salomon : correspondances, rapports (1936).

\section{NOTES}

1. Association pour l'avancement des sciences, Saint-Étienne, t. 2, 1897, p. 245.

2. James Jackson fils est l'un des témoins de la cérémonie de mariage entre Nicolas-Alexis Massenet et Adélaïde Royer de Marancour en 1830.

3. DESCREUX Denis, Notices Biographiques Stéphanoises, Saint-Étienne, Imprimerie Théolier, 1868, p.231-232.

4. Ibidem, p.231-232.

5. JACKSON William, James Jackson et ses Fils, 1883, p.118-119.

6. Ibidem, p.118-119.

7. Ibid., p.122.

8. L'entreprise Jacob Holtzer et Cie est l'entreprise du beau-père de Pierre-Frédéric Dorian. Ce dernier dirige d'ailleurs cette société.

9. VALSERRES Jacques, Les Industries de la Loire, Saint-Étienne, Robin, 1862, p.127-128.

10. AD Haute-Loire, Fonds Dorian-Holtzer-Jackson, 1 A 1 , constitution de l'affaire : coupure de presse (1856).

11. JACKSON William, James Jackson..., op. cit., 1883, p.124.

12. VALSERRES Jacques, Les industries..., op. cit., 1862, p.131.

13. AD Haute-Loire, $9 \mathrm{M} \mathrm{15}$, inspection du travail, établissement soumis à l'inspection du travail : liste de la commune de Pont-Salomon (16 août 1897).

14. AD Haute-Loire, fonds Dorian-Holtzer-Jackson, $1 \mathrm{~J}$ 14, répertoire des commettants de l'entreprise : grand livre (1858-1952).

15. AD Haute-Loire, fonds Dorian-Holtzer-Jackson, $1 \mathrm{~J}$ 36, importation-exportation : tableau, correspondance, rapport (1860-1949).

16. Voir ROJAS Luc, L'industrie stéphanoise: de l'espionnage industriel à la veille technologique (1700-1950), Paris, l'Harmattan, 2009.

17. AD Haute-Loire, fonds Dorian-Holtzer-Jackson, 3 A 1, écrits historiques : lettre de M. Adolf Reile (16 septembre 1950).

18. VALSERRES Jacques, Les Industrie..., op. cit., p.132.

19. AD Haute-Loire, $9 \mathrm{M} \mathrm{7}$, statistiques industrielles du département de la Haute-Loire (1856-1868).

20. AD Haute-Loire, fonds Dorian-Holtzer-Jackson, 5 D 1, rapport de stage sur l'installation hydro-électrique de la Semène et son utilisation par l'usine (1922 et 1946).

21. AD Haute-Loire, fonds Dorian-Holtzer-Jackson, 3 D 2, énergie électrique: plans, correspondance, rapport (1925-1981). 
22. AD Haute-Loire, fonds Dorian-Holtzer-Jackson, E 2, Enquêtes sur le parc de machines outils : rapport (1960).

23. AD Haute-Loire, fonds Dorian-Holtzer-Jackson, $\mathrm{K} 8$, études des temps de travail, prix de revient : rapport sur l'état de consommation pour fabrication des faux (1857-1858).

24. AD Haute-Loire, fonds Dorian-Holtzer-Jackson, $\mathrm{K}$ 8, études des temps de travail, prix de revient : rapport sur la fabrication de chaque type de faux (1932-1933).

25. VALSERRES Jacques, Les industries..., op. cit., 1862, p.131.

26. AD Haute-Loire, fonds Dorian-Holtzer-Jackson, $\mathrm{K} 8$, études des temps de travail, prix de revient : rapport sur la fabrication de chaque type de faux (1932-1933).

27. AD Haute-Loire, fonds Dorian-Holtzer-Jackson, $\mathrm{K} 8$, études des temps de travail, prix de revient : étude sur le temps de travail (1967).

28. FOURIER Charles, Le Nouveau Monde Industriel et Sociétaire, Librairie de l'école sociétaire, 1845, p.139-141.

29. En 1912 le site est complété par la construction d'un « château » pour le directeur.

30. VALSERRES Jacques, L'industrie..., op. cit., 1862, p.125.

31. AD Haute-Loire, Fonds Dorian-Holtzer-Jackson, 1 D 3, ventes de terrains: actes notariés (1877-1956).

32. Député à partir de 1863 puis ministre en 1870.

33. AD Haute-Loire, Fonds Dorian-Holtzer-Jackson, dossiers individuels, Fleury Binachon: dossiers, correspondance....

34. AD Haute-Loire, Fonds Dorian-Holtzer-Jackson, 2 D 2, logements de l'entreprise : état des lieux, inventaires, correspondance (1954-1985).

35. AD Haute-Loire, Fonds Dorian-Holtzer-Jackson, 2 D 1, jardins ouvriers: plans, correspondance, bon d'attribution (1930-1985).

36. FOURIER Charles, Le nouveau monde..., op. cit., 1845, p.473.

37. $\mathrm{AD}$ Haute-Loire, Fonds Dorian-Holtzer-Jackson, $2 \mathrm{D}$ 1, bâtiments sociaux appartenant à l'entreprise : dossiers sur l'église et le presbytère (1983-1985).

38. AD Haute-Loire, Fonds Dorian-Holtzer-Jackson, 2 A 3, dossiers individuels : statuts de la société de secours mutuels fondé par les ouvriers de la société des fabriques des faux de PontSalomon (1866).

39. AD Haute-Loire, Fonds Dorian-Holtzer-Jackson, 5 L 2, Cercle des ouvriers : statuts, règlements, situation comptable (1881-1967).

40. AD Haute-Loire, Fonds Dorian-Holtzer-Jackson, 5 L 2, Cercle des ouvriers : statuts, règlements, situation comptable (1881-1967).

41. AD Haute-Loire, $10 \mathrm{M} 36$, syndicats professionnels, Pont-Salomon : correspondances, rapports (1936).

42. AD Haute-Loire, Fonds Dorian-Holtzer-Jackson, 2 D 1, construction du bureau de poste: correspondance, plan, devis, bail (1937).

43. AD Haute-Loire, Fonds Dorian-Holtzer-Jackson, 3 D 2, énergie électrique: plans correspondance, rapport (1925-1981).

44. Anonyme, Les Établissements Dorian-Holtzer-Jackson et Cie, circa 1970, p.6.

45. AD Haute-Loire, Fonds Dorian-Holtzer-Jackson, 3 D 3, adduction d'eau: plans, schémas, rapports (1933).

46. AD Haute-Loire, Fonds Dorian-Holtzer-Jackson, 2 D 1, Bâtiments sociaux, résumé des travaux effectués dans la salle des fêtes : rapport (1949).

47. AD Haute-Loire, Fonds Dorian-Holtzer-Jackson, 5 L 4, cinéma: note de service, registre comptabilité... (1950-1970). 


\section{RÉSUMÉS}

Dès la fin des années 1830, les métallurgistes du bassin industriel stéphanois cherchent à diversifier leur activité. En 1838 certains d'entre-eux, Alexis Massenet notamment, arrivent dans la vallée de la Semène en Haute-Loire afin de mettre en place de petites unités de production de faux et de faucilles. De grands entrepreneurs tels Jackson et Holtzer le rejoignent rapidement. Ce mouvement prend une autre dimension avec le projet de Pierre-Frédéric Dorian en 1857. Influencé par le fouriériste Victor Considérant, qui avait fondé une colonie industrielle «La Réunion » au Texas quelques années auparavant, Dorian érige à Pont-Salomon une usine appelée «L'Alliance » qui va rapidement agréger les différents ateliers disposés le long de la Semène et ne former qu'une seule entité devenant ainsi un véritable village usine avec ses logements ouvriers, son église, son école, son système social...

At the end of the 1830's, the metallurgists of Saint-Etienne industrial district, try to diversify their activity. In 1838, Alexis Massenet come into the valley of Semène in Haute-Loire to build a small production units of scythes and sickles. He is quickly joined by others metallurgists like Jackson and Holtzer. This movement takes another dimension with Pierre-Frédéric Dorian's project in 1857. Strongly influenced by the fouriériste Victor Considérant, Dorian sets up in PontSalomon a factory named "The Alliance" which is quickly going to aggregate the various arranged workshops along the Semène valley. This entity becomes a factory town with its worker's housings, its church, its school and its social system....

\section{INDEX}

Mots-clés : histoire des techniques, France, XIXe siècle, colonie industrielle, Fouriérisme, métallurgie, utopie, modernité

Keywords : History of technology, France, XIXth century, company town, Fourier, metallurgical industry, utopia, modernity

\section{AUTEUR}

\section{LUC ROJAS}

Luc Rojas est chercheur associé à l'UMR CNRS 5600 (EVS-ISTHME). Ses préoccupations scientifiques portent sur les mutations et les changements technologiques entre les XVIII ${ }^{e}$ et XX siècles. À travers ce champ d'investigation, il aborde de nombreux thèmes de recherche dont les principaux sont : les pratiques et les pensées des ingénieurs civils, la circulation de l'information et des idées techniques et les traces et mémoires de la civilisation industrielle. Depuis quelques années, il concentre ses travaux sur l'organisation du travail et des entreprises notamment sur la pensée et l'action d'Henri Fayol. 